\title{
AN ALGORITHM FOR SOLVING INVERSE PROBLEMS OF HEAT AND MASS TRANSPORT IN AGRICULTURAL PRODUCTS
}

\author{
Jerzy Weres, Zbigniew Dworecki, Mariusz Loboda \\ Department of Agricultural Engineering, Agricultural University of Poznań \\ Wojska Polskiego 50, 60-627 Poznań, Poland \\ e-mail: weres@au.poznan.pl
}

\begin{abstract}
An algorithm was developed for solving transient, three-dimensional, quasi-linear, inverse problems of heat and mass transport in nonhomogeneous agricultural products of irregular geometry. A numerical approach consisted of an optimization technique and a generalized finite element method, with the use of isoparametric, curvilinear, 3D-space elements, and a three-point recurrence scheme in time, absolutely stable, with iterative procedure to deal with the quasi-linearity of equations. A computer program was developed and the method was applied to estimate the moisture diffusion coefficient, and then to predict heat and moisture transport in individual corn kernels during drying.
\end{abstract}

\section{Introduction}

Proper understanding of heat and mass transport in complex agricultural products is an essential ingredient in the advancement of food processing systems. Therefore, fast and reliable computer methods for predicting such processes as heating, drying, and cooling of biomaterials are of particular importance.

Accuracy of predictions depends on adequate representation of the process parameters in the mathematical model. In case of heat and mass transport in agricultural products, it has not been fully determined which of the factors responsible for the process should be considered in the model. It has generally been accepted that the effective moisture transport coefficient is the most critical quantity affecting accuracy of predictions in case of drying agricultural products [4, 5, 6, 9], Its value is highly dependent on the product moisture content, changes during drying, and also manifests significant differences for the product components. Such nonhomogeneity is the most significant factor in the first and middle periods of dry ing, whereas the geometric irregularity of the product is the most pronounced factor in the final period [9, 11],

Due to the complexity of biomaterials and the interactions which occur between the product and the surrounding fluid, direct determination of some coefficients is cumbersome and often leads to erroneous results. Thus, an indirect approach was examined in this study to estimate unknown coefficient values, based on the inverse heat and mass transport concept [1, 2, 3, 8, 10], optimization techniques [7], and the structural mathematical model for the corresponding direct problems of heat and mass transport [9, 11],

The objectives of this study were to develop a numerical algorithm for solving transient, three-dimensional, quasi-linear, inverse problems of heat and mass transport in nonhomogeneous agricultural products of irregular geometry, and to estimate, using measured and predicted moisture content values, the moisture diffusion coefficient in the thin-layer drying of corn. 


\section{Development of an algorithm}

\subsection{Mathematical model}

The mathematical structural model of heat and mass transport can be represented as the following system of coupled quasi-linear differential equations of heat conduction and moisture diffusion with the initial and boundary conditions of the first and third kind $[6,9]$ :

$$
\begin{gathered}
\frac{\partial}{\partial t} T-\nabla\left(\frac{\lambda}{\rho c} \nabla T\right)-\alpha_{v} \frac{\partial}{\partial t} M=0, \quad(x, t) \in \Omega \times\left(0, t_{F}\right], \\
\frac{\partial}{\partial t} M-\nabla\left(D_{m} \nabla M\right)-\nabla\left(D_{m T} \nabla T\right)=0, \quad(x, t) \in \Omega \times\left(0, t_{F}\right], \\
T(x, 0)=T_{0}(x), \quad M(x, 0)=M_{0}(x), \quad(x) \in \Omega, \\
T(x, t)=T_{s}(x), \quad M(x, t)=M_{e}(x), \quad(x, t) \in \partial \Omega^{I} \times\left(0, t_{F}\right], \\
\lambda n \nabla T+h_{c T}\left(T-T_{\infty}\right)=0, \quad D_{m} n \nabla M+h_{c m}\left(M-M_{e}\right)=0, \quad(x, t) \in \partial \Omega^{I I I} \times\left(0, t_{F}\right] .
\end{gathered}
$$

The operational model was developed by applying the finite element approximation to Eqns (1-5) with the use of isoparametric, curvilinear, three-dimensional elements (rectangular prisms as parent elements), and a three-point recurrence scheme in time, absolutely stable, with an iterative procedure to deal with the quasi-linearity of equations [9, 11], Procedures controlling the solution accuracy, stability and susceptibility to oscillations, and the computational efficiency were also developed. The final operational model is given as:

- Three-point recurrence scheme composed of algebraic equations:

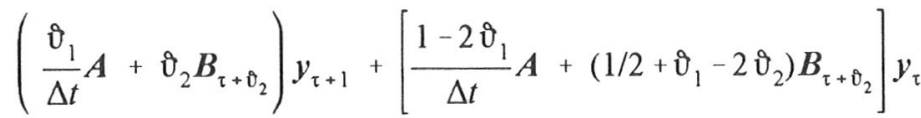

$$
\begin{aligned}
& +\left[\frac{\mathrm{v}_{1}-1}{\Delta t} A+\left(1 / 2-\hat{\mathrm{v}}_{1}+\mathrm{v}_{2}\right) B_{\tau+\mathrm{v}_{2}}\right] y_{\tau-1}+\varphi_{\tau+\mathrm{v}_{2}}=0
\end{aligned}
$$


where

$$
\begin{aligned}
& y_{\tau}^{2 k-1} \equiv T^{k}\left(x^{k}, t_{\tau}\right), \quad y_{\tau}^{2 k} \equiv M^{k}\left(x^{k}, t_{\tau}\right), \quad k=1, \ldots, N N, \\
& B_{\tau+\hat{\theta}_{2}} \equiv B\left(y_{\tau+\hat{\theta}_{2}}, t_{\tau+\hat{\theta}_{2}}\right), \quad \varphi_{\tau+\hat{\theta}_{2}} \equiv \varphi\left(y_{\tau+\hat{0}_{2}}, t_{\tau+\hat{\theta}_{2}}\right), \quad y_{\tau+\hat{\theta}_{2}}=y_{\tau}+\hat{\theta}_{2}\left(y_{\tau}-y_{\tau-1}\right), \\
& t_{\tau+\hat{\theta}_{2}}=t_{\tau}+\hat{\theta}_{2} \Delta t, \quad 2 \Delta t=t_{\tau+1}-t_{\tau-1}, \quad \tau=1, \ldots, N T-1 .
\end{aligned}
$$

- Two-point starting scheme for the first time interval.

- Set of data representing conditions of the process.

- Empirical equations to calculate the equilibrium moisture content and the moisture diffusion coefficients for the grain kernel components.

Material nonhomogeneity and geometric irregularity of a product (a corn kernel in the example investigated) were considered as shown in Fig. 1. Photographs of kernel sections were taken, and then curvilinear 3D elements were related to corresponding nodes and material components in the global orthocartesian system of coordinates. The analysis
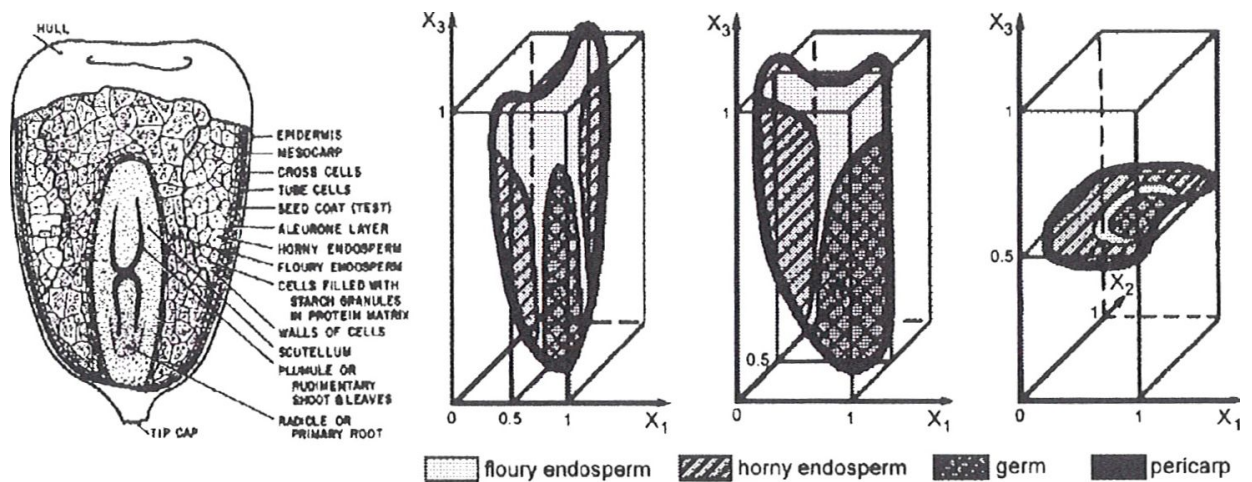

Fig. 1. The representation of nonhomogeneity and geometric irregularity of an agricultural product (corn kernel) by means of three sections against a background of the basic solid.

of convergence, stability, susceptibility of solutions to oscillations, and computational efficiency was carried out. Due to the stiffness of the problem, the extreme eigenvalues of the global matrix were estimated, the vanishing times for the solution components were determined, and accordingly two phases of solution were introduced, differing in time intervals. To reduce a very strong oscillatory disturbance of solutions due to the abrupt change of the boundary condition at the initial instant of the process, a finer grid was applied. 
The operational structural model presented in this section covers significant factors which have been disregarded in most of other models, as reviewed in [5, 9], Accuracy of predictions depends critically on those factors, and simplification of the model leads to erroneous results, as depicted in Fig. 2.

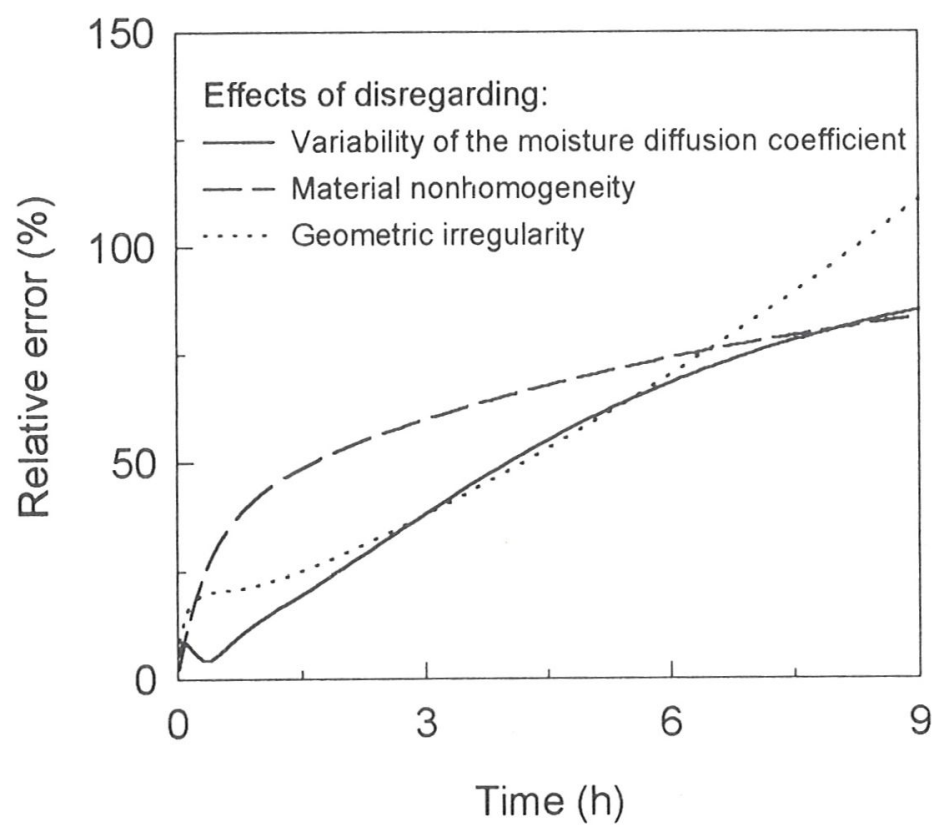

Fig. 2. Effects of disregarding variability of the moisture diffusion coefficient, material nonhomogeneity and geometric irregularity on the accuracy of predictions for moisture transport in individual corn kernels.

2.2 The algorithm for solving inverse heat and mass transport problems

The final algorithm was developed on the basis of an inverse problem approach, the operational model for solving direct problems presented in Section 2.1, and the quasiNewton optimization technique. The algorithm, given below, can be used for solving transient, three-dimensional, quasi-linear, inverse problems of heat and mass transport in nonhomogeneous agricultural products of irregular geometry. 
Read the set of data items, including:

measured temperature and/or moisture content values at selected locations in the interior of a product and selected time instants,

the initial guess for the selected coefficient subject to estimation the thermal conductivity, the moisture diffusion coefficient, the convective heat transfer coefficient or the convective mass transfer coefficient).

Start the iterative instruction of finding minimum of the objective function with respect to the coefficient values subject to estimation by the quasi-Newton method until the assumed accuracy or the maximum number of iterations is reached.

Determine the space and time distributions of the temperature and/or the moisture content by the PES module (Parabolic Equation Solver).

Compute the objective function, defined as the sum of the squares of the difference between the measured and predicted temperature and/or moisture content values.

Write the current results (values of the iteration index, the objective function and the coefficient estimated) to the database file.

Increase the iteration index value.

End the iterative instruction of finding minimum of the objective function when the assumed accuracy or the maximum number of iterations is reached.

Write the final results to appropriate database files.

\section{Method}

The algorithm for solving inverse problems of heat and mass transport in agricultural products was applied to solve a problem of thin-layer drying of corn. Empirical data on moisture content changes in dried corn were collected experimentally as described in [9]. The operational structural model presented in Section 2.1 was used to predict the corresponding moisture content changes numerically. The results were compared, and the objective function was computed as the sum of the squares of the difference between the measured and predicted moisture content values. The objective function was minimized with respect to the coefficient subject to estimation by the quasi-Newton method. The gradient of the objective function was also determined to control accuracy of the optimization procedure. The estimated values of the coefficient (the moisture diffusion coefficient in this case) were used to predict the corn kernel drying behavior, and the proposed method was validated by comparing final numerical predictions (moisture content changes), averaged over the grain kernel domain, to an additional set of experimental data.

A computer program (Inverse Problem Solver) was designed and coded in Fortran. The IPS program included appropriate modules for solving direct heat and mass transport problems (Parabolic Equation Solver), for computing the objective function, and for minimizing this function with respect to the estimated coefficient. Numerical simulations were performed for 480 curvilinear isoparametric 8-node hexahedral elements, 693 no- 
des, and 300 time intervals, with an iterative procedure due to quasi-linearity of equations.

\section{Discussion of results and conclusion}

Application of the developed algorithm to solve an inverse problem of thin-layer drying of corn, with the moisture diffusion coefficient as the coefficient sought, resulted in an increase in accuracy of prediction. Moisture content changes in dried corn kernels, computed for uncertain values of the moisture diffusion coefficient given in literature [9] and validated by experimental data, showed the global relative error of $10.6 \%$. That value was reduced to $6.5 \%$ when the proposed algorithm was used to estimate the moisture diffusion coefficient.

The algorithm presented in this study can be used to solve accurately and effectively inverse problems of heat and mass transport in complex agricultural products. It can handle transient character of the process, dependence of the transport coefficients on temperature and moisture content, material nonhomogeneity and geometric irregularity of three-dimensional objects, and natural (third kind) boundary conditions. Estimation of unknown coefficient values is possible for the thermal conductivity, the moisture diffusion coefficient, and the convective heat and moisture transfer coefficients in the boundary layer.

If experimental data representing process parameters are questionable, and thus a solution to a direct heat and mass transport problem is inaccurate, implementation of the inverse problem concept discussed in this study is recommended. In particular, if values of the basic process parameters such as the thermal conductivity, the moisture diffusion coefficient, and the convective heat and moisture transfer coefficients in the boundary layer are uncertain, the algorithm can be very useful.

\section{Acknowledgments}

This work was supported by the Polish Committee for Scientific Research as the 8T11F01011 research grant. Numerical experiments were performed on computers of the Poznań Supercomputing and Networking Center.

\section{References}

1. Alifanov, O.M. 1994. Inverse heat transfer problems. Berlin, Springer-Verlag.

2. Anger, G. 1990. Inverse problems in differential equations. New York, Plenum.

3. Ciałkowski, M. 1996. Selected methods and algorithms for solving inverse problem for the heat conduction equation (in Polish). Poznań, WPP.

4. Irudayaraj, J. and $\mathrm{Wu}, \mathrm{Y}$. 1994. Finite element analysis of coupled heat, mass, and pressure transfer in porous biomaterials. Numerical Heat Transfer, Part A 26, 337-350. 
5. Oliveira, L.S. and Haghighi, K. 1997. Finite element modeling of grain drying. In: Mathematical modeling and numerical techniques in drying technology (eds. I. Turner and A S. Mujumdar), 309-338. New York, Marcel Dekker, Inc.

6. Pabis S. 1982. Theory of convective drying of agricultural products (in Polish). Warszawa, PWRiL.

7. Press, W.H., Teukolsky, S.A., Vetterling, W.T., and Flannery, B P. 1992. Numerical Recipes. Cambridge, Cambridge University Press.

8. Tarantola, A. 1987. Inverse Problem Theory. Methods for Data Fitting and Model Parameter Estimation. Amsterdam, Elsevier.

9. Weres, J. and Jayas, D.S. 1994. Effects of corn kernel properties on predictions of moisture transport in the thin-layer drying of corn. Transactions of the ASAE 37(5), $1695-1705$.

10. Weres, J., Jayas, D.S., and Ryniecki, A. 1994. An inverse heat transfer method for the estimation of convective heat transfer coefficient for foods. ASAE Paper No. 94-3036. St. Joseph, MI, Am. Soc. of Agric. Eng.

11. Weres, J. 1997. Finite element analysis of heat and mass transport in biological materials. Proc. XIII Polish Conf. on Computer Methods in Mechanics, vol. 4, 1387-1392. Poznań, Polish Association for Computational Mechanics.

\section{List of symbols}

$\mathbf{A}, \mathbf{B}=$ coefficient matrices of the global set of algebraic equations,

c $\quad=$ specific heat $\left(\mathrm{J} \cdot \mathrm{kg}^{-1} \cdot \mathrm{K}^{-1}\right)$,

$\mathrm{D}_{\mathrm{m}} \quad=$ moisture diffusion coefficient $\left(\mathrm{m}^{2} \bullet \mathrm{s}^{-1}\right)$,

$\mathrm{D}_{\mathrm{mT}}=$ moisture thermodiffusion coefficient $\left(\mathrm{m}^{2} \cdot \mathrm{K} \cdot \mathrm{s}^{-1}\right)$,

$\mathrm{h}_{\text {ст }}=$ convective heat transfer coefficient in the boundary layer of the domain $\Omega$ $\left(\mathrm{W} \cdot \mathrm{m}^{-2} \cdot \mathrm{K}^{-1}\right)$,

$\mathrm{h}_{\mathrm{cm}}=$ convective moisture transfer coefficient in the boundary layer of the domain $\Omega$ $\left(\mathrm{m} \cdot \mathrm{s}^{-1}\right)$,

$\mathrm{M}=$ moisture content at point $\mathrm{x} \in \Omega$ and time $\mathrm{t} \in\left(0, \mathrm{t}_{\mathrm{F}}\right]$ (decimal, dry basis),

$\mathrm{M}_{0} \quad=$ moisture content at point $\mathrm{x} \in \Omega$ and time $\mathrm{t}=0$ (decimal, dry basis),

$\mathrm{M}_{\mathrm{e}} \quad=$ equilibrium moisture content determined either at point $\mathbf{x} \in \partial \Omega$ or at points outside the boundary layer of the domain $\Omega$ at time $\mathrm{t} \in\left(0, \mathrm{t}_{\mathrm{F}}\right]$ (decimal, dry basis),

NT $=$ number of time intervals,

n $\quad=$ unit vector normal to the surface $\partial \Omega$, directed outward,

$\mathrm{t} \quad=$ time $(\mathrm{s})$,

$\mathrm{t}_{\mathrm{F}}=$ instant limiting time of the process from the right side (s),

$\mathrm{T}=$ temperature at point $\mathrm{x} \in \Omega$ and time $\mathrm{t} \in\left(0, \mathrm{t}_{\mathrm{F}}\right](\mathrm{K})$,

$\mathrm{T}_{0} \quad=$ temperature at point $\mathrm{x} \in \Omega$ and time $\mathrm{t}=0(\mathrm{~K})$,

$\mathrm{T}, \quad=$ air temperature at points outside the boundary layer of at time $\mathrm{t} \in\left(0, \mathrm{t}_{\mathrm{F}}\right](\mathrm{K})$, 


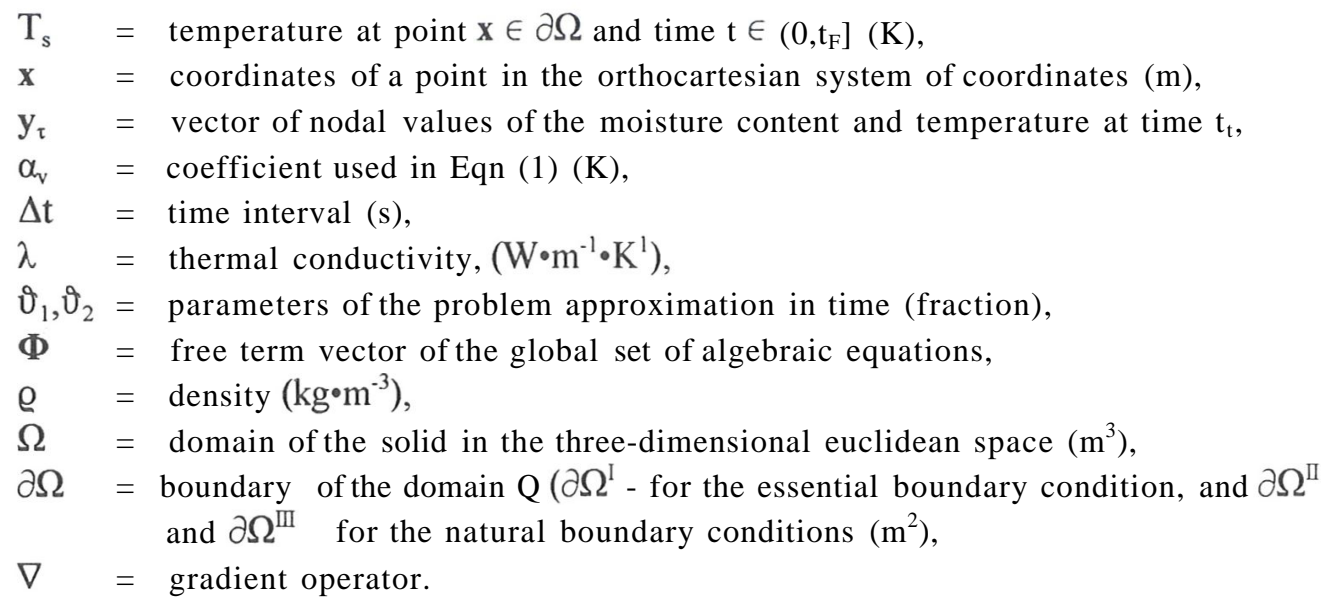

